Check for updates

Cite this: J. Mater. Chem. A, 2019, 7, 14753

Received 23rd March 2019

Accepted 20th May 2019

DOI: 10.1039/c9ta03131f

rsc.li/materials-a

\section{Highly efficient and stable inverted perovskite solar cells using down-shifting quantum dots as a light management layer and moisture-assisted film growth $\dagger$}

\author{
Mohammad Mahdi Tavakoli, (D) *ab Hadi Tavakoli Dastjerdi, ${ }^{c}$ Daniel Prochowicz, (D) d \\ Pankaj Yadav, (D) ${ }^{e}$ Rouhollah Tavakoli, ${ }^{a}$ Michael Saliba ${ }^{f}$ and Zhiyong Fan (D)*gh
}

Stability is one of the key challenges for perovskite solar cells (PSCs) to become an industrial reality. In this work, we present two novel strategies to significantly improve the stability of PSCs using environmental humidity and down-shifting quantum dots (QDs). We first improve the crystallinity of double-cation Cs/ FA perovskite by annealing films in $40 \%$ relative humidity $(\mathrm{RH})$. Our results reveal that the grain size, carrier lifetime and crystallinity of perovskite films are enhanced drastically due to the humidity. Next, we apply a thin layer of CdSe/CdS quantum dots (QDs) on the back of the device to convert high energy UV light into lower energy visible wavelengths. Using this modification, we improve not only the UV stability but also the device performance of the PSCs. Based on these modifications, we achieve an inverted PSC with a maximum power conversion efficiency (PCE) of $20.7 \%$. Moreover, our modified device shows a great operational stability under continuous illumination after $300 \mathrm{~h}$ with only $15 \%$ PCE loss. We also examine the UV stability of our devices and find that the modified PSC retains $79 \%$ of its initial PCE after $150 \mathrm{~h}$ under continuous illumination outperforming the reference device.

\section{Introduction}

Organometallic halide perovskites have an $\mathrm{ABX}_{3}$ structure, where $\mathrm{A}$ is cesium (Cs), methylammonium (MA) or formamidinium (FA), B is $\mathrm{Pb}$ or $\mathrm{Sn}$, and $\mathrm{X}$ is $\mathrm{Cl}, \mathrm{Br}$ or $\mathrm{I}^{\mathbf{1 - 3}}$ These compounds emerge as attractive light harvesting materials due to their intriguing optoelectronic properties such as high mobility, long carrier life time, band gap tunability, great absorption and ease of fabrication. In the past few years,

${ }^{a}$ Department of Materials Science and Engineering, Sharif University of Technology, 14588 Tehran, Iran

${ }^{b}$ Department of Electrical Engineering and Computer Science, Massachusetts Institute of Technology, Cambridge, MA 02139, USA. E-mail: mtavakol@mit.edu

'Department of Materials Science and Engineering, Massachusetts Institute of Technology, Cambridge, MA 02139, USA

${ }^{d}$ Institute of Physical Chemistry, Polish Academy of Sciences, Kasprzaka 44/52, 01-224 Warsaw, Poland

${ }^{e}$ Department of Solar Energy, School of Technology, Pandit Deendayal Petroleum University, Gandhinagar-382 007, Gujarat, India

${ }^{f}$ Adolphe Merkle Institute, Chemins des Verdiers 4, CH-1700 Fribourg, Switzerland ${ }^{g}$ Department of Electronic and Computer Engineering, The Hong Kong University of Science and Technology, Clear Water Bay, Kowloon, Hong Kong SAR, China. E-mail: eezfan@ust.hk

${ }^{h}$ HKUST-Shenzhen Research Institute, No. 9 Yuexing First RD, South Area, Hi-tech Park, Nanshan, Shenzhen 518057, China

$\dagger$ Electronic supplementary information (ESI) available. See DOI: $10.1039 / \mathrm{c} 9 \operatorname{ta} 03131 \mathrm{f}$ perovskite solar cells (PSCs) showed tremendous progress with a certified PCE that is over $24 \% .^{4-9}$ Besides the further improvement of photovoltaic performance, long-term device stability is a key challenge for the commercialization of PSCs. ${ }^{\mathbf{1 0 1 1}}$ The main reported strategies to improve stability include compositional engineering, ${ }^{12-14}$ interface modification, ${ }^{15,16}$ passivation techniques, ${ }^{17,18}$ using water-repellent materials ${ }^{19-22}$ and device packaging via encapsulation methods. ${ }^{2324}$ These techniques enhance the device performance and stability by reducing the recombination sites and improving the quality of PSCs. ${ }^{25-27}$ Among the above approaches, the positive effects of humidity either in perovskite precursors ${ }^{28}$ or during the annealing step $^{29}$ on the improvement of perovskite crystallinity have been reported by many researchers in this field. In general, a perovskite compound decomposes in a highly humid environment; however, an optimum level of moisture can have a positive impact on the optical properties and the morphology of perovskite films. ${ }^{30}$ For example, Contreras-Bernal et $a .^{31}$ studied the effect of the humidity level during the fabrication process on the stability of PSCs and found that the as-prepared perovskite films under controlled humid conditions below 50\% RH show better stability in a humid environment.

Another drawback of perovskite materials is the presence of organic components that can decompose upon exposure to high energy UV light..$^{32-34}$ One promising strategy to tackle this issue 
is the application of a protective layer on the top of a PSC that blocks the UV photons from reaching the perovskite material and converts them into lower energy visible photons. ${ }^{35-37}$ In this context, luminescent down shifting materials such as fluorinated photopolymers, ${ }^{38}$ complex compounds such as lanthanide luminescence and europium complexes, ${ }^{35,39}$ and QDs $^{\mathbf{4 0 - 4 3}}$ demonstrate potential to improve the light absorption and current density and reduce the degradation of PSCs under UV light.

In this work, we demonstrate effective strategies to improve the stability and performance of inverted PSCs based on a Cs/FA double A-cation composition via annealing perovskite films in a humid environment and application of a luminescent downshifting quantum dot (QD) layer. We find that the annealing of perovskite films in an ambient air atmosphere with $40 \% \mathrm{RH}$ effectively increases the grain size and film quality leading to an improved carrier lifetime. Further deposition of a core-shell CdSe/CdS QD thin film on the back side of a PSC successfully suppresses the degradation of the perovskite material under irradiation of UV light. As a result, the inverted PSC yields in a high PCE of $20.70 \%$ and improved stability under the conditions of continuous UV illumination for $150 \mathrm{~h}$. These results open new possibilities for the development of efficient and stable PSCs.

\section{Results and discussion}

Here, we use a double A-cation Cs/FA perovskite composition (see details in the ESI $\dagger$ ) as an absorber layer embedded in an inverted architecture. The perovskite film was deposited using the anti-solvent method ${ }^{27}$ and annealed at $110{ }^{\circ} \mathrm{C}$ for $40 \mathrm{~min}$ in a nitrogen glovebox (the reference) and in a dry air box with $40 \%$ $\mathrm{RH}$ (the ambient air sample) for comparison. Fig. 1a and b show the schematics of the perovskite films deposited on ITO glass annealed inside the nitrogen glovebox and in ambient air, respectively. Top-view scanning electron microscopy (SEM) images of both samples reveal that the average grain size increases significantly from 320 to $760 \mathrm{~nm}$ by annealing the perovskite film under ambient air conditions (Fig. 1c and d). This indicates that moisture has a positive effect on the improvement of the grain size and crystallinity of perovskite films, resulting in a reduction of the formed grain boundaries. ${ }^{\mathbf{4 4 5}}$ This result is also confirmed by atomic force microscopy (AFM), as shown in Fig. 1e and f. Moreover, the surface roughness of the perovskite films is reduced from $31 \pm 8 \mathrm{~nm}$ to $25 \pm 6 \mathrm{~nm}$ after annealing in ambient air as compared to the reference sample (see Fig. $\mathrm{S} 1 \dagger$ ).

The optical properties of the perovskite films are examined by UV-visible absorption and photoluminescence (PL) measurements. As shown in Fig. 2a, the optical absorption and emission of the perovskite film annealed in ambient air are stronger than those observed for the reference, indicating a significant effect of humidity on the perovskite crystallinity and grain size. Moreover, we find that the band edges of the absorption spectra slightly red shift when the film is annealed in ambient air. Similarly, the PL spectrum shows a correspondingly small red shift with respect to the reference film. (a)

(b)
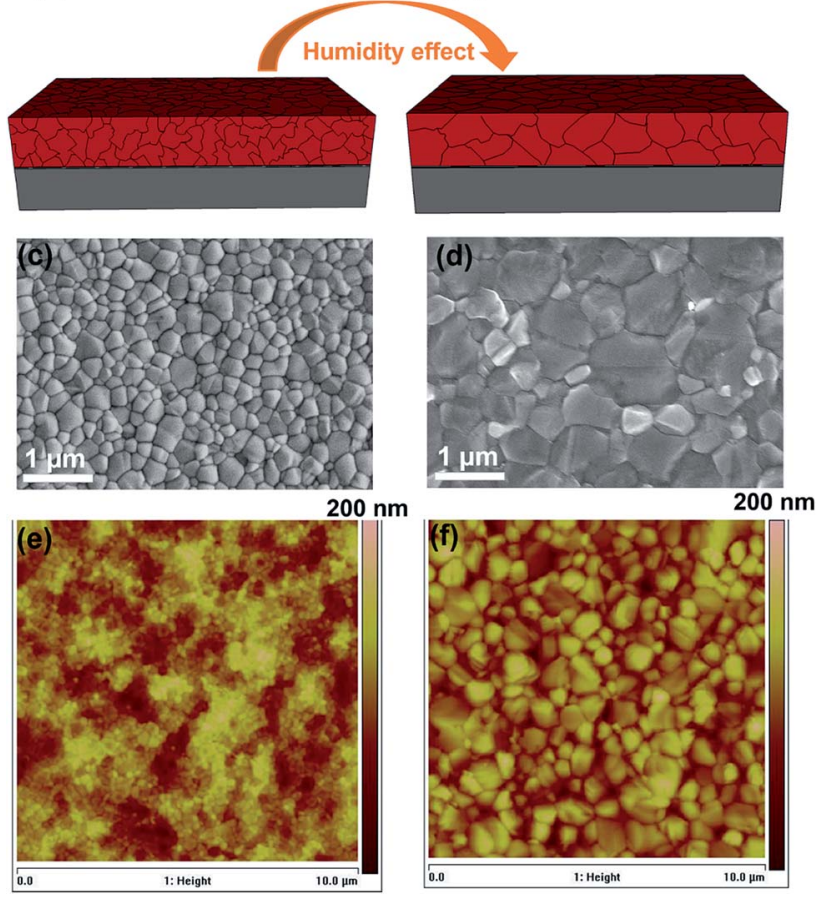

Fig. 1 Schematic representations of the perovskite films after annealing at $110^{\circ} \mathrm{C}(\mathrm{a})$ in a nitrogen and (b) humid atmosphere ( 40\% $\mathrm{RH}$ ). Top-view SEM and AFM images of the perovskite films annealed inside a nitrogen-filled glovebox ( $c$ and e) and in ambient air ( $d$ and f), respectively.

The red shift in both UV-vis and PL spectra might be caused by the larger grain size, consistent with the SEM images. ${ }^{46-48}$ To further determine the carrier lifetimes of the resulting perovskite films, time-resolved PL (TRPL) measurements were performed. The enhancement of the carrier lifetime from 3.39 to $7.36 \mathrm{~ns}$ is observed for the perovskite film annealed in ambient air, which could be due to its larger grain size and better crystallinity (see Fig. 1). The fitting parameters of TRPL data are listed in Table S1.† Fig. 2c shows the X-ray diffraction (XRD) patterns of the perovskite films annealed in the different environments. Both films exhibit a similar structure with the perovskite peaks at $\sim 14.0^{\circ}$ and $\sim 28.0^{\circ}$ originating from the (110) and (220) planes of the perovskite phase ${ }^{45}$ and the intensity of these diffraction peaks is more than that for the film annealed in ambient air indicating its higher crystallinity. In addition, in both samples the characteristic diffraction peaks specific to $\mathrm{PbI}_{2}$ and non-perovskite yellow phase $\delta$-FAPbI 3 were not observed. These results demonstrate that the annealing of the perovskite films in ambient air facilitates its crystal growth.

To study the impact of the humidity effect on the resulting photovoltaic properties, we fabricate devices with an inverted architecture of ITO/NiO/perovskite/C60/BCP/Ag. Fig. 3a shows the cross-sectional SEM image of an inverted PSC device based on the perovskite film fabricated in ambient air. The device is comprised of indium-doped tin oxide (ITO) as a transparent electrode, a $40 \mathrm{~nm}$-thick nickel oxide (NiO) layer as a hole transporting layer (HTL), a $450 \mathrm{~nm}$-thick perovskite film as an absorber layer, a $23 \mathrm{~nm}$ thick C60 layer as an electron 
(a)

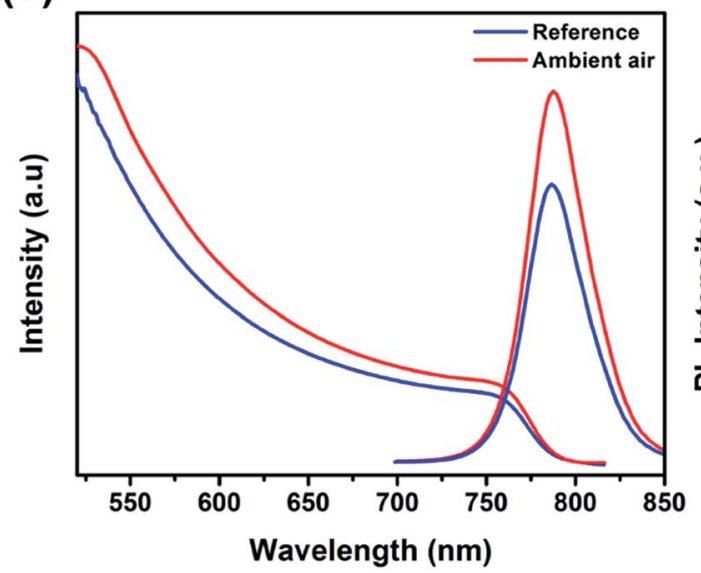

(b)

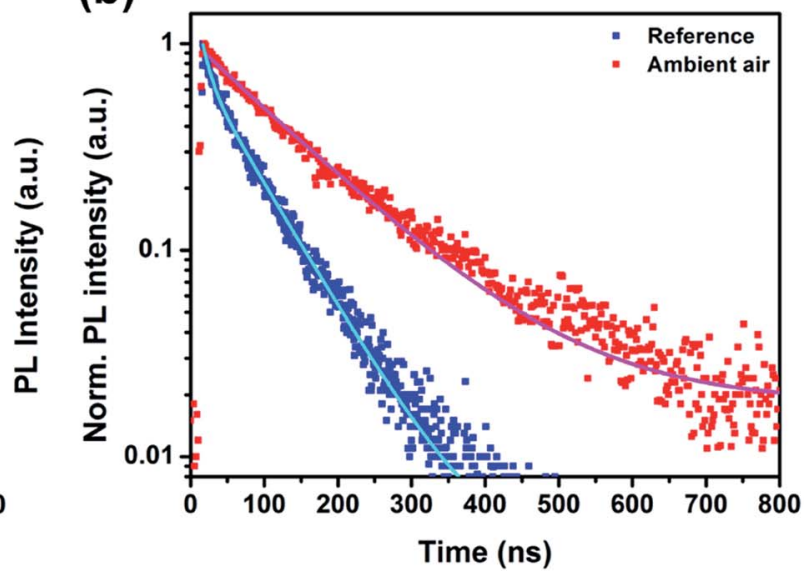

(c)

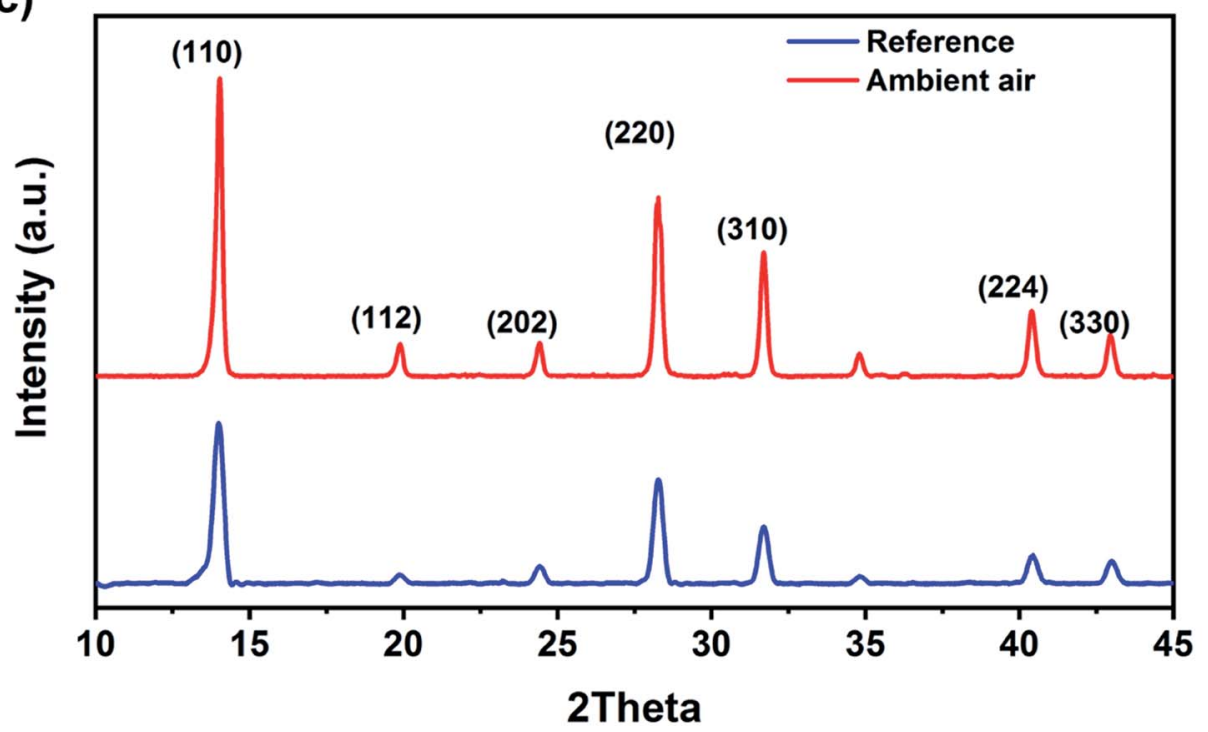

Fig. 2 Characterization of the perovskite films annealed in a nitrogen glovebox and ambient air. (a) UV-visible and photoluminescence spectra, (b) time-resolved photoluminescence (TRPL) spectra and (c) XRD patterns.

transporting layer (ETL), an $8 \mathrm{~nm}$-thick layer of bathocuproine (BCP) as a buffer layer, and a silver (Ag) electrode with a thickness of $100 \mathrm{~nm}$. Fig. 3b shows the current densityvoltage $(J-V)$ curves of the best performing PSC devices based on perovskite films fabricated under nitrogen and ambient air conditions. The summary of photovoltaic (PV) parameters is given in Table 1. The reference device shows an open circuit voltage $\left(V_{\mathrm{oc}}\right)$ of $1125 \mathrm{mV}$, a short circuit current density $\left(J_{\mathrm{sc}}\right)$ of $22.1 \mathrm{~mA} \mathrm{~cm}^{-2}$, a fill factor of $75.6 \%$ and a PCE of $18.8 \%$. However, the ambient air device has a superior photovoltaic performance with a $V_{\mathrm{oc}}$ of $1141 \mathrm{mV}$, a $J_{\mathrm{sc}}$ of $23.05 \mathrm{~mA} \mathrm{~cm} \mathrm{~cm}^{-2}$, a fill factor of $77 \%$, and a high PCE of $20.2 \%$. These results indicate that the humidity effect has a great impact on all PV parameters for the investigated PSC. By controlling the level of moisture during the annealing step, the quality of the perovskite film can be improved drastically as substantiated by the presented characterization technique. We also track the maximum power point (MPP) of the best-performing devices as shown in Fig. 3c. The devices based on perovskite films annealed in the nitrogen glovebox and ambient air show MPPs of $18.4 \%$ and $20.1 \%$ over $60 \mathrm{~s}$, confirming their performance stability under light. The statistics of the collected PV parameters for the corresponding devices are shown in Fig. S2. $\uparrow$ The average values of all PV parameters are improved drastically as compared to the reference cells. To further investigate the humidity effect on the $J_{\text {sc }}$ of these devices, we measured their external quantum efficiency (EQE). The ambient air device exhibits a higher EQE (more than $85 \%$ ) over the entire spectrum as compared to the reference cell, resulting in an integrated $J_{\mathrm{sc}}$ of $21.87 \mathrm{~mA} \mathrm{~cm}^{-2}$ higher than that of the reference device with $20.92 \mathrm{~mA} \mathrm{~cm} \mathrm{~cm}^{-2}$ (Fig. 3d). These results indicate a good consistency of the integrated $J_{\mathrm{sc}}$ with the $J_{\mathrm{sc}}$ values obtained from the $J-V$ curves. The higher current density of the ambient air device can be ascribed to the lower recombination sites due to the better crystallinity and morphology of the perovskite. 
(a)

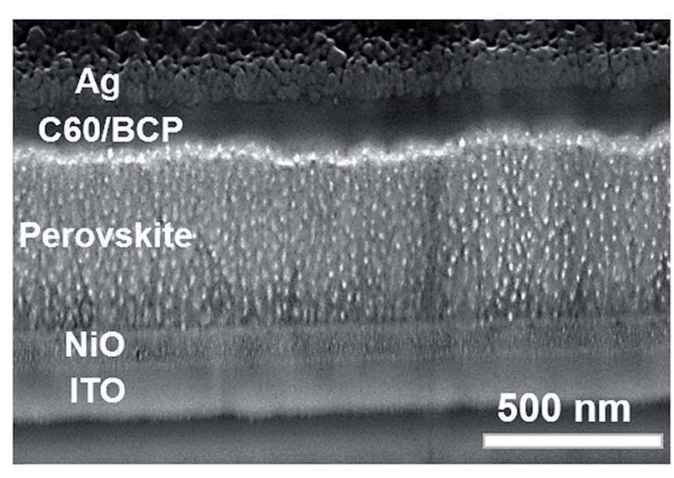

(c)

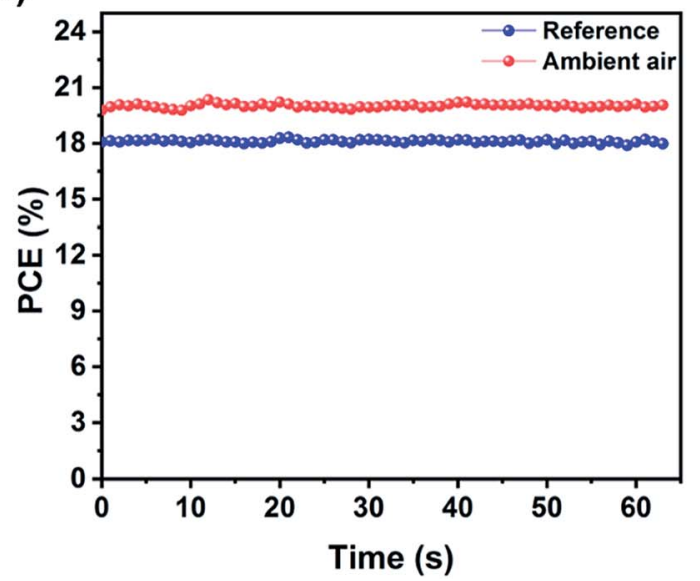

(b)

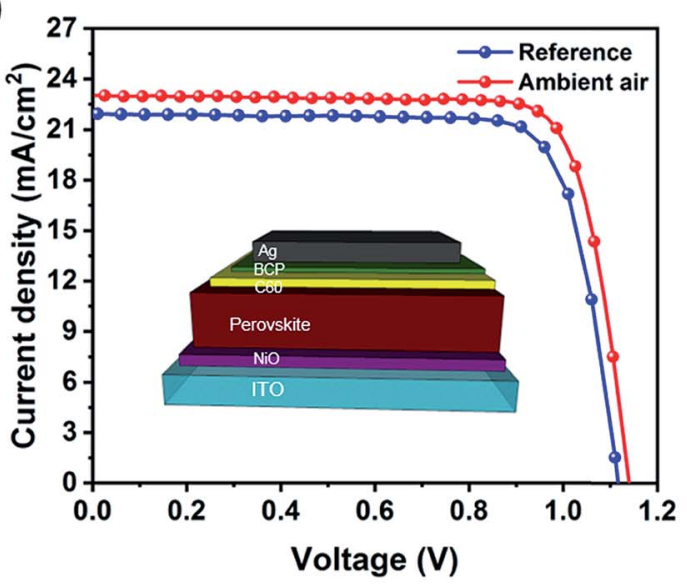

(d)

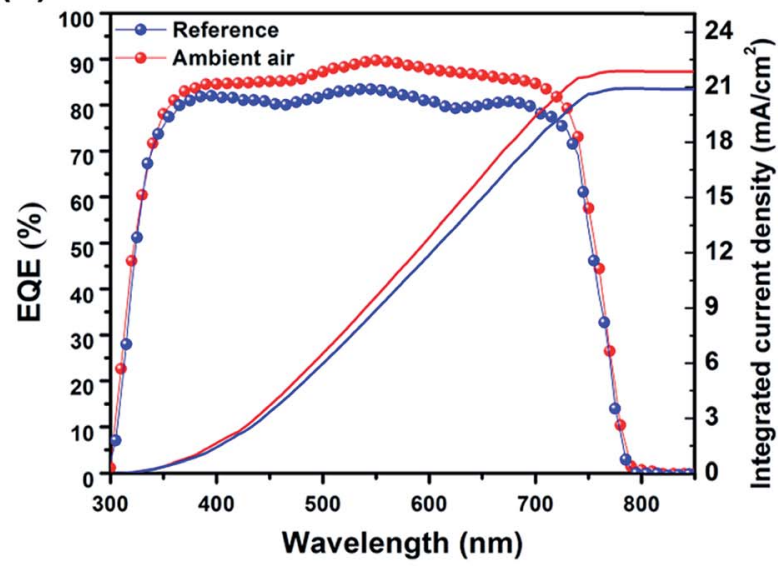

Fig. 3 (a) Cross-sectional SEM image, (b) J-V curves, (c) maximum power point tracking and (d) EQE spectra of the PSC devices based on perovskite films annealed in the nitrogen glovebox and ambient air.

Table 1 Figures of merit for the PSC devices based on perovskite films annealed in a nitrogen glovebox and ambient air

\begin{tabular}{llllll}
\hline Device & $V_{\mathrm{oc}}(\mathrm{mV})$ & $J_{\mathrm{sc}}\left(\mathrm{mA} \mathrm{cm}^{-2}\right)$ & $\mathrm{FF}(\%)$ & PCE (\%) & MPP (\%) \\
\hline Reference & 1125 & 22.10 & 75.6 & 18.8 & 18.4 \\
Ambient air & 1141 & 23.05 & 77 & 20.2 & 20.1
\end{tabular}

Fig. S3†shows the statistics of hysteresis indices (HIs) for the investigated devices. Our results show an average $\mathrm{HI}$ of $1.2 \%$ for the devices based on perovskite films annealed in ambient air, which is lower than that observed for reference devices (1.6\%). This result shows the impact of perovskite crystallinity on the mitigation of hysteresis behavior in the investigated PSCs.

In order to study the effect of humidity on the PV parameters of the PSCs, electrochemical impedance spectroscopy (EIS) was employed. Fig. $\mathrm{S} 4 \uparrow$ illustrates the Nyquist plots of the reference device and modified PSC under dark conditions. The series resistance $\left(R_{\mathrm{S}}\right)$ and recombination resistance $\left(R_{\mathrm{Rec}}\right)$ of the corresponding PSCs were estimated by fitting the Nyquist plots using the equivalent circuit shown in the inset image of Fig. S4. $\dagger$ Our results indicate that the ambient air PSC has a lower $R_{\mathrm{S}}(18.6 \Omega)$ as compared to the reference cell $(25.2 \Omega)$, which explains the higher FF of the modified PSC. ${ }^{9}$ Additionally, the $R_{\text {Rec }}$ of the reference cell and modified PSC is 306 and $415 \Omega$ $\mathrm{cm}^{-2}$, respectively. It is clear that the modified PSC has a larger recombination resistance, i.e., lower recombination than that of the reference device, resulting in a PSC with a higher $V_{\text {oc. }}{ }^{30}$ This effect is originated from the quality of the perovskite film annealed in ambient air with a larger grain size than that of the reference sample (Fig. 1d and f). Consequently, the modified PSC has lower grain boundaries and recombination sites, which results in a higher $V_{\text {oc }}$ as well as PCE. ${ }^{\mathbf{9} 30}$

Apart from the high efficiency, the stability of PSCs under illumination, heat, moisture and also UV light irradiation remains a key challenge for the commercialization of PSCs. ${ }^{3}$ To address the photostability issue, we deposited a thin layer of core-shell CdSe/CdS QDs (with 85\% PL quantum yield (PLQY)) as a down-shifting layer on the back of the device and optimize its thickness by controlling the QD concentration in hexane from 0 to $2.0 \mathrm{wt} \%$. Essentially, CdSe/CdS QDs possess a wide absorption band in the UV region and can convert high energy photons into low energy photons. This procedure should result in more efficient light absorption and higher device stability. Fig. 4a shows the emission of the QD layer with different dot concentrations. As seen, this layer shows a strong red emission, 
(a)
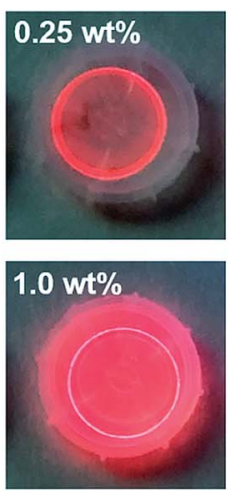

(c)
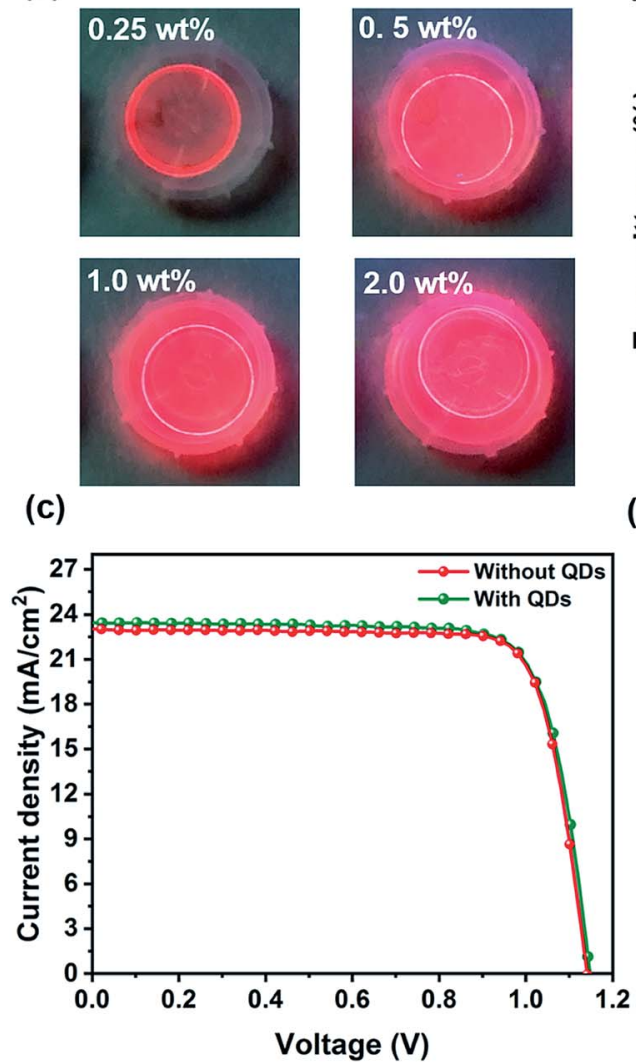

(b)

(d)
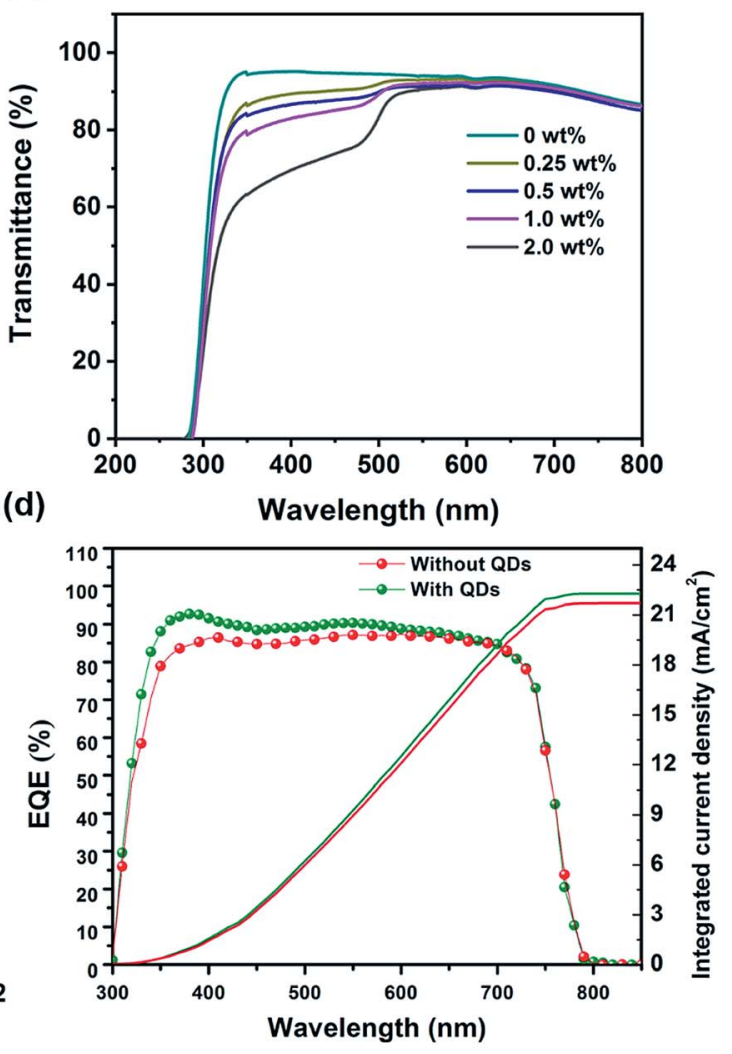

Fig. 4 (a) Photographs of the emission of CdSe/CdS QDs deposited on glass with different thicknesses under UV light and (b) transmittance spectra of the corresponding QD films. (c) J-V curves and (d) EQE spectra of PSCs without and with a down-shifting QD layer.

which is intensified by increasing the thickness of the QD layer. The steady-state PL spectrum of CdSe/CdS QDs shows a PL peak position at $620 \mathrm{~nm}$ (Fig. S5†). Fig. S6† shows that upon increasing the thickness of the QD layer (using more concentrated solutions), the PL intensity of the QD layer is increased, indicating a stronger down-shifting effect. The transmittance spectra of the QD layer with different thicknesses are shown in Fig. 4b. It is clear that the QD layer mostly blocks the light from 300 to $500 \mathrm{~nm}$. In the next step, we try to find an optimum thickness of this layer, which will have a superior impact on the final device performance. The PV results for this optimization are listed in Table S2.† We find that a QD concentration of $1.0 \mathrm{wt} \%$ is the optimum value, increasing the current density as well as blocking the high energy photons. Fig. 4 c depicts the $J-V$ curves of the best PSC devices annealed in ambient air without and with the QD layer. Table 2 summarizes the collected PV parameters of the corresponding devices. By applying the QD layer on the back of the device, the $J_{\mathrm{sc}}$ is increased from 23.1 to

Table 2 Figures of merit for the PSC devices without and with the down-converting QD layer

\begin{tabular}{lllll}
\hline Device & $V_{\mathrm{oc}}(\mathrm{mV})$ & $J_{\mathrm{sc}}\left(\mathrm{mA} \mathrm{cm}^{-2}\right)$ & $\mathrm{FF}(\%)$ & PCE (\%) \\
\hline Without QDs & 1141 & 23.1 & 76.2 & 20.08 \\
With QDs & 1143 & 23.6 & 76.8 & 20.70
\end{tabular}

$23.6 \mathrm{~mA} \mathrm{~cm}^{-2}$ with a slight enhancement in the $V_{\mathrm{oc}}$ from 1141 to $1143 \mathrm{mV}$, resulting in a PCE of 20.7\%, which is higher than that of the PSC device without the QD layer (20.08\%). Moreover, the obtained high PCE is among the highest efficiencies reported for inverted PSCs. ${ }^{49}$ Fig. $4 \mathrm{~d}$ demonstrates the EQE curves of the corresponding devices without and with the QD layer. It is evident that the EQE of the QD based device is higher in the high energy photon range, i.e. the UV region, due to the downshifting properties of the QD layer. In fact, the UV light in this sample is converted to the visible range (around $620 \mathrm{~nm}$ ) and absorbed in the perovskite film, which results in higher EQE at shorter wavelengths. Consequently, the integrated $J_{\mathrm{sc}}$ calculated from the EQE curve of the PSC with the QD layer is estimated to be $22.3 \mathrm{~mA} \mathrm{~cm}^{-2}$, which is $0.6 \mathrm{~mA} \mathrm{~cm}{ }^{-2}$ higher than that of the reference sample. These results are in agreement with the $J_{\mathrm{sc}}$ values obtained from the $J-V$ curves. For the devices without the down-shifting QD layer, the high energy photons, with wavelengths of $<\sim 400 \mathrm{~nm}$, get significantly absorbed in the FTO electrode and $\mathrm{NiO}_{x} \mathrm{HTL}$, before they can reach the perovskite layer. On the other hand, even for the small percentage of incident high energy photons that could reach the perovskite layer, their contribution to carrier generation would be very small due to high levels of thermalization in the perovskite layer. During the EQE measurement of the PSCs with the QD layer, the high energy photons that would otherwise contribute very little to carrier generation for the reference PSC are 


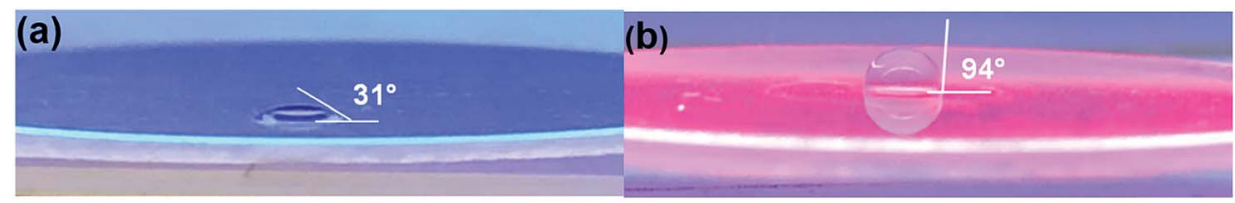

(c)

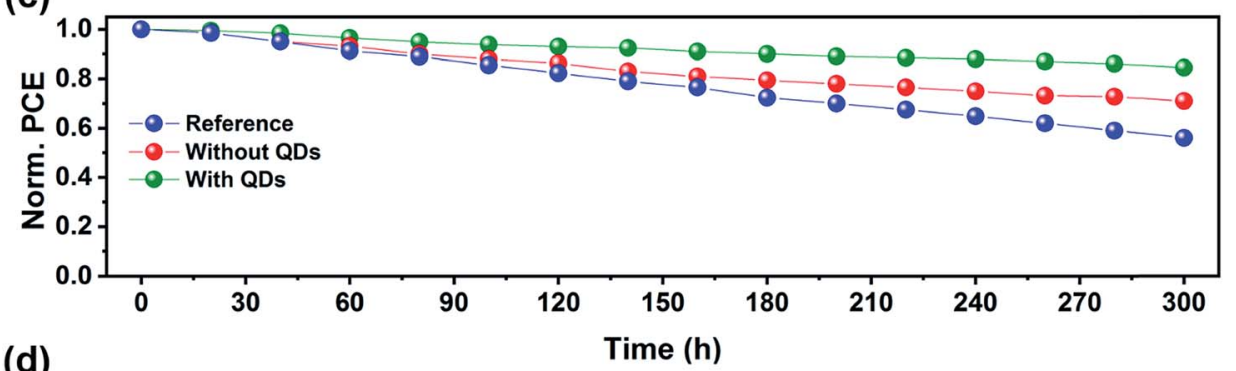

(d)

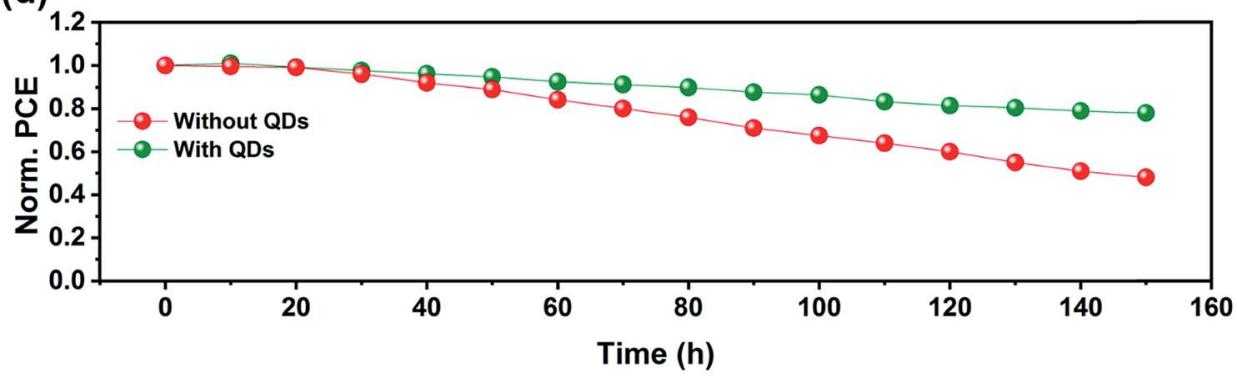

Fig. 5 Contact angle measurement of (a) pure glass and (b) the down-shifting layer on glass using a water droplet. Stability test of the PSC devices (c) under continuous illumination for $300 \mathrm{~h}$ and (d) under UV light for $150 \mathrm{~h}$.

absorbed by the QD layer and get re-emitted at longer wavelengths. The re-emitted photons at longer wavelengths are highly absorbed by the perovskite layer and effectively contribute to carrier generation resulting in improved EQE in the UV range and slightly improved $\mathrm{EQE}$ in the visible region.

Fig. 5a and b show the contact angle (CA) of a water droplet on pure glass and a QD layer deposited on glass under UV illumination, respectively. Interestingly, by applying the QD layer on the surface of glass, i.e., the back of the PSC device, the CA is increased from $31^{\circ}$ to $94^{\circ}$. This result indicates that the QD layer can work as a protection layer for PSCs in a solar farm against moisture. To study the effect of down-shifting materials on the device stability, we perform stability tests under continuous illumination and UV light irradiation. Fig. 5c shows the stability results of the PSCs under continuous illumination for $300 \mathrm{~h}$ in a nitrogen environment. The PSC device annealed in ambient air with the QD layer shows the best stability with only $15 \%$ PCE loss after $300 \mathrm{~h}$ illumination. In turn, the analogous device without the QD layer has a $28 \%$ PCE loss, which is more stable than the reference PSC with a $44 \%$ PCE drop. These results highlight the effect of humidity on the perovskite crystallinity as well as the device stability. We further examine the stability of PSCs without and with QD layers under continuous UV light irradiation. As shown in Fig. 5d, the protected device retains $79 \%$ of its initial PCE after exposure to continuous UV light over $150 \mathrm{~h}$, and is more stable than the reference device without QD layers (retaining only $48 \%$ of its initial PCE value). This enhancement stems from the QD down-shifting layer, which blocks the UV light and improves the device stability.

\section{Conclusions}

In summary, we develop two strategies to improve the device performance and stability of inverted PSCs based on a Cs/FA double A-cation composition, i.e. annealing in an ambient environment with $40 \% \mathrm{RH}$ and applying a down-shifting layer on the back of the PSC. We demonstrate that ambient air annealing of perovskite films improves the grain size and crystallinity leading to a longer carrier lifetime as compared to perovskite films annealed in a nitrogen glovebox. Based on this strategy, we improve the device performance from $18.8 \%$ to $20.2 \%$ and enhance its operational stability. Additionally, we show that the application of a CdSe/CdS QD layer on the back of the PSC as a down-shifting layer results in a higher $J_{\mathrm{sc}}$ and PCE, with a maximum efficiency of $20.7 \%$ and better light stability. Moreover, the device with the QD layer maintains $79 \%$ of its initial PCE value after exposure to continuous UV illumination for $150 \mathrm{~h}$, a clear improvement compared to the reference sample that loses $52 \%$ of its initial PCE.

\section{Experimental section}

\section{Device fabrication}

ITO glass was patterned using zinc powder and diluted $\mathrm{HCl}$ solution and then cleaned in the following solutions for $20 \mathrm{~min}$ 
using ultrasonic treatment, respectively: Triton X100 (1\% vol in deionized (DI) water), DI water, acetone, and ethanol. The surface of the cleaned ITO glass was treated using UV ozone before the deposition of NiO as an HTL. A precursor solution of $\mathrm{NiO}(1 \mathrm{M})$ was prepared by dissolving nickel(II) nitrate hexahydrate $\left(\mathrm{Ni}\left(\mathrm{NO}_{3}\right)_{2} \cdot 6 \mathrm{H}_{2} \mathrm{O}\right.$, Sigma Aldrich) in ethylene glycol with added ethylenediamine (Sigma Aldrich) and lithium acetate. Then, this solution was spin-coated on the substrates at $3000 \mathrm{rpm}$ for $100 \mathrm{~s}$, followed by annealing at $300{ }^{\circ} \mathrm{C}$ in air for $1 \mathrm{~h}$.

After treating the surface of the NiO layer using UV ozone, a precursor solution of $\mathrm{Cs} / \mathrm{FA}$ perovskite containing $\mathrm{PbI}_{2}$ (1.26M, TCI), FAI (1.08M, Dyesol), and $\mathrm{CsCl}(0.12 \mathrm{M}, \mathrm{TCI})$ in a mixed solvent of DMF and DMSO (4/1 vol\%) was spin coated in two steps: $1000 \mathrm{rpm}$ for $10 \mathrm{~s}$ and $6000 \mathrm{rpm}$ for $20 \mathrm{~s}$ (ramp rate: $2000 \mathrm{rpm} \mathrm{s}^{-1}$ ). During spin-coating, $60 \mu \mathrm{L}$ of chlorobenzene (CB) (on a 0.5 inch $^{2}$ substrate) anti-solvent was added dropwise on the film, $10 \mathrm{~s}$ before the end of the spinning. 1-adamantylamine hydrochloride (ADAHCl) was dissolved in $\mathrm{CB}(3 \mathrm{mg}$ $\mathrm{mL}^{-1}$ ) for the passivation purpose as reported previously. Afterward, the films were annealed at $110^{\circ} \mathrm{C}$ for $40 \mathrm{~min}$ in either a nitrogen glovebox or ambient air with $40 \%$ RH. After annealing, the films were transferred to a thermal evaporator and $\mathrm{C} 60(23 \mathrm{~nm}), \mathrm{BCP}(8 \mathrm{~nm})$, and $\mathrm{Ag}(100 \mathrm{~nm})$ were evaporated on the perovskite film, as an ETL, buffer layer, and electrode, respectively. For the deposition of the core-shell structure of CdSe/CdS QDs (Sigma Aldrich), solutions with different concentrations in hexane $(0,0.25,0.5,1.0$, and $2.0 \mathrm{wt} \%)$ were prepared and deposited at $3000 \mathrm{rpm}$ for $30 \mathrm{~s}$ to obtain layers with different thicknesses.

\section{Film characterization}

The morphology of the perovskite films was studied using scanning electron microscopy (SEM, ZEISS Merlin), X-ray diffraction (XRD, Bruker D8 X-ray Diffractometer (USA)), and atomic force microscopy (AFM, NanoScope IIIa/Dimension 3100). The optical properties of the films were analyzed by UV-visible (Varian Cary 5) and photoluminescence (PL, a Fluorolog 322 (Horiba Jobin Ybon Ltd)) measurements. The PL excitation was at $\lambda=460 \mathrm{~nm}$. For lifetime measurement, a picosecond pulsed diode laser (EPL-405, with an excitation wavelength of $\lambda=405 \mathrm{~nm}$ and a pulse width of $49 \mathrm{ps)}$ was employed and the data were fitted using an exponential equation $\left(I(t)=a_{\mathrm{i}} \exp \left(-t / \tau_{\mathrm{i}}\right)\right)$, where $a_{\mathrm{i}}$ and $\tau_{\mathrm{i}}$ are the amplitude and the lifetime of each term, respectively.

\section{Device measurement}

All devices were measured by using a digital source meter (Keithley model 2400, USA) and a $450 \mathrm{~W}$ xenon lamp (Oriel, USA). The simulated light was filtered using a Schott K113 Tempax sunlight filter (Präzisions Glas \& Optik GmbH, Germany). For $J-V$ measurement, the voltage scan rate and the dwell time were set to $10 \mathrm{mV} \mathrm{s}^{-1}$ and $15 \mathrm{~s}$, respectively. To simulate AM $1.5 \mathrm{G}$ conditions, the light intensity was adjusted to $1000 \mathrm{~W} \mathrm{~m}^{-2}$. The hysteresis indices of PSCs were calculated using the following formula: hysteresis index $=\left(\left(\mathrm{PCE}_{\text {backward }}-\right.\right.$
$\left.\mathrm{PCE}_{\text {forward }} / \mathrm{PCE}_{\text {backward }}\right) \times 100$. The external quantum efficiency of the devices was recorded by using a commercial apparatus (Arkeo-Ariadne, Cicci Research s.r.l.) with a 300 Watts xenon lamp.

The stability measurement (including UV stability) was performed in a nitrogen glovebox. The devices were kept under simulated standard AM $1.5 \mathrm{G}$ conditions using an array of white LED lamps and the maximum power point (MPP) was measured over time. For UV stability, a UV lamp with $100 \mathrm{~mW} \mathrm{~cm}^{-2}$ was used. EIS measurement was performed using Autolab with a frequency range from $200 \mathrm{mHz}$ to1 $\mathrm{MHz}$.

\section{Conflicts of interest}

There is no conflict to declare.

\section{Acknowledgements}

This work was supported by the National Natural Science Foundation of China (Project No. 51672231), Shen Zhen Science and Technology Innovation Commission (Project No. JCYJ20170818114107730) and Hong Kong Research Grant Council (General Research Fund Project No. 16237816). H. T. D. acknowledges postdoctoral fellowship support from the Natural Sciences and Engineering Research Council (NSERC) of Canada.

\section{Notes and references}

1 N. G. Park, M. Grätzel, T. Miyasaka, K. Zhu and K. Emery, Nat. Energy, 2016, 1, 16152.

2 J. W. Lee, H. S. Kim and N. G. Park, Acc. Chem. Res., 2016, 49, 311-319.

3 M. M. Tavakoli, S. M. Zakeeruddin, M. Grätzel and Z. Fan, Adv. Mater., 2018, 30, 1705998.

4 N. J. Jeon, H. Na, E. H. Jung, T. Y. Yang, Y. G. Lee, G. Kim, H. W. Shin, S. I. Seok, J. Lee and J. A. Seo, Nat. Energy, 2018, 3, 682 .

5 National Center for Photovoltaics (NCPV) at the National Renewable Energy Laboratory (NREL) efficiency chartwww.nrel.gov/pv/assets/images/efficiency-chart.png.

6 M. M. Tavakoli, W. Tress, J. V. Milić, D. Kubicki, L. Emsley and M. Grätzel, Energy Environ. Sci., 2018, 11, 3310.

7 N. J. Jeon, J. H. Noh, W. S. Yang, Y. C. Kim, S. Ryu, J. Seo and S. I. Seok, Nature, 2015, 517, 476.

8 E. H. Jung, N. J. Jeon, E. Y. Park, C. S. Moon, T. J. Shin, T. Y. Yang, J. H. Noh and J. Seo, Nature, 2019, 567, 511.

9 D. Prochowicz, M. M. Tavakoli, A. Solanki, T. W. Goh, K. Pandey, T. C. Sum, M. Saliba and P. Yadav, J. Mater. Chem. A, 2018, 6, 14307-14314.

10 M. M. Tavakoli, P. Yadav, D. Prochowicz, M. Sponseller, A. Osherov, V. Bulović and J. Kong, Adv. Energy Mater., 2019, 1803587.

11 D. Prochowicz, R. Runjhun, M. M. Tavakoli, P. Yadav, M. Saski, A. Q. Alanazi, D. J. Kubicki, Z. Kaszkur, S. M. Zakeeruddin, J. Lewiński and M. Grätzel, Chem. Mater., 2019, 31, 1620-1627. 
12 D. Prochowicz, M. M. Tavakoli, A. Kalam, R. D. Chavan, S. Trivedi, M. Kumar and P. Yadav, J. Mater. Chem. A, 2019, 7, 8218-8225.

13 M. M. Tavakoli, L. Gu, Y. Gao, C. Reckmeier, J. He, A. L. Rogach, Y. Yao and Z. Fan, Sci. Rep., 2015, 5, 14083.

14 D. Prochowicz, P. Yadav, M. Saliba, D. J. Kubicki, M. M. Tavakoli, S. M. Zakeeruddin, J. Lewiński, L. Emsley and M. Grätzel, Nano Energy, 2018, 49, 523-528.

15 M. M. Tavakoli, R. Tavakoli, Z. Nourbakhsh, A. Waleed, U. S. Virk and Z. Fan, Adv. Mater. Interfaces, 2016, 3, 1500790.

16 M. M. Tavakoli, R. Tavakoli, S. Hasanzadeh and M. H. Mirfasih, J. Phys. Chem. C, 2016, 120, 19531-19536.

17 X. Zheng, B. Chen, J. Dai, Y. Fang, Y. Bai, Y. Lin, H. Wei, X. C. Zeng and J. Huang, Nat. Energy, 2017, 2, 17102.

18 M. M. Tavakoli, D. Bi, L. Pan, A. Hagfeldt, S. M. Zakeeruddin and M. Grätzel, Adv. Energy Mater., 2018, 8, 1800275.

19 S. F. Leung, Q. Zhang, M. M. Tavakoli, J. He, X. Mo and Z. Fan, Small, 2016, 12, 2536-2548.

20 Y. Lin, Z. Xu, D. Yu, L. Lu, M. Yin, M. M. Tavakoli, X. Chen, Y. Hao, Z. Fan, Y. Cui and D. Li, ACS Appl. Mater. Interfaces, 2016, 8, 10929-10936.

21 Q. Lin, L. Lu, M. M. Tavakoli, C. Zhang, G. C. Lui, Z. Chen, X. Chen, L. Tang, D. Zhang, Y. Lin and P. Chang, Nano Energy, 2016, 22, 539-547.

22 M. M. Tavakoli, K. H. Tsui, Q. Zhang, J. He, Y. Yao, D. Li and Z. Fan, ACS Nano, 2015, 9, 10287-10295.

23 A. Waleed, M. M. Tavakoli, L. Gu, Z. Wang, D. Zhang, A. Manikandan, Q. Zhang, R. Zhang, Y. L. Chueh and Z. Fan, Nano Lett., 2016, 17, 523-530.

24 Y. Han, S. Meyer, Y. Dkhissi, K. Weber, J. M. Pringle, U. Bach, L. Spiccia and Y. B. Cheng, J. Mater. Chem. A, 2016, 3, 81398147.

25 M. Yang, Z. Li, M. O. Reese, O. G. Reid, D. H. Kim, S. Siol, T. R. Klein, Y. Yan, J. J. Berry, M. F. van Hest and K. Zhu, Nat. Energy, 2017, 2, 17038.

26 P. Yadav, S. H. Turren-Cruz, D. Prochowicz, M. M. Tavakoli, K. Pandey, S. M. Zakeeruddin, M. Grätzel, A. Hagfeldt and M. Saliba, J. Phys. Chem. C, 2018, 122, 15149-15154.

27 M. M. Tavakoli, M. Saliba, P. Yadav, P. Holzhey, A. Hagfeldt, S. M. Zakeeruddin and M. Grätzel, Adv. Energy Mater., 2019, 9, 1802646.

28 X. Gong, M. Li, X. B. Shi, H. Ma, Z. K. Wang and L. S. Liao, Adv. Funct. Mater., 2015, 25, 6671-6678.

29 H. Zhou, Q. Chen, G. Li, S. Luo, T. B. Song, H. S. Duan, Z. Hong, J. You, Y. Liu and Y. Yang, Science, 2014, 345, 542-546.
30 M. M. Tavakoli, R. Tavakoli, P. Yadav and J. Kong, J. Mater. Chem. A, 2019, 7, 679-686.

31 L. Contreras-Bernal, C. Aranda, M. Valles-Pelarda, T. T. Ngo, S. Ramos-Terrón, J. J. Gallardo, J. Navas, A. Guerrero, I. Mora-Seró, J. Idígoras and J. A. Anta, J. Phys. Chem. C, 2015, 122, 5341-5348.

32 M. M. Tavakoli, F. Giordano, S. M. Zakeeruddin and M. Grätzel, Nano Lett., 2018, 18, 2428-2434.

33 C. Chen, H. Li, J. Jin, X. Chen, Y. Cheng, Y. Zheng, D. Liu, L. Xu, H. Song and Q. Dai, Adv. Energy Mater., 2018, 7, 1700758.

34 M. M. Tavakoli, P. Yadav, R. Tavakoli and J. Kong, Adv. Energy Mater., 2018, 8, 1800794.

35 C. W. Kim, T. Y. Eom, I. S. Yang, B. S. Kim, W. I. Lee, Y. S. Kang and Y. S. Kang, Sci. Rep., 2017, 7, 6849.

36 L. Jiang, W. Chen, J. Zheng, L. Zhu, L. E. Mo, Z. Li, L. Hu, T. Hayat, A. Alsaedi, C. Zhang and S. Dai, ACS Appl. Mater. Interfaces, 2017, 9, 26958-26964.

37 D. Zhou, D. Liu, G. Pan, X. Chen, D. Li, W. Xu, X. Bai and H. Song, Adv. Mater., 2017, 29, 1704149.

38 F. Bella, G. Griffini, J. P. Correa-Baena, G. Saracco, M. Grätzel, A. Hagfeldt, S. Turri and C. Gerbaldi, Science, 2016, 354, 203-206.

39 Z. Hosseini and T. Ghanbari, RSC Adv., 2018, 8, 3150231509.

40 A. Tayyebi, M. M. Tavakoli, M. Outokesh, A. Shafiekhani and A. Simchi, Ind. Eng. Chem. Res., 2015, 54, 7382-7392.

41 Q. Wang, X. Zhang, Z. Jin, J. Zhang, Z. Gao, Y. Li and S. F. Liu, ACS Energy Lett., 2017, 2, 1479-1486.

42 M. B. De La Mora, O. Amelines-Sarria, B. M. Monroy, C. D. Hernández-Pérez and J. E. Lugo, Sol. Energy Mater. Sol. Cells, 2017, 165, 59-71.

43 M. M. Tavakoli, A. Simchi, Z. Fan and H. Aashuri, Chem. Commun., 2016, 52, 323-326.

44 Z. Chu, M. Yang, P. Schulz, D. Wu, X. Ma, E. Seifert, L. Sun, X. Li, K. Zhu and K. Lai, Nat. Commun., 2017, 8, 2230.

45 M. M. Tavakoli, Q. Lin, S. F. Leung, G. C. Lui, H. Lu, L. Li, B. Xiang and Z. Fan, Nanoscale, 2016, 8, 4276-4283.

46 Y. Kanemitsu, J. Mater. Chem. C, 2017, 5, 3427-3437.

47 M. M. Tavakoli, A. Waleed, L. Gu, D. Zhang, R. Tavakoli, B. Lei, W. Su, F. Fang and Z. Fan, Nanoscale, 2017, 9, 58285834.

48 A. Waleed, Q. Zhang, M. M. Tavakoli, S. F. Leung, L. Gu, J. He, X. Mo and Z. Fan, Sci. Bull., 2016, 61, 86-91.

49 H. Chen, F. Ye, W. Tang, J. He, M. Yin, Y. Wang, F. Xie, E. Bi, X. Yang, M. Grätzel and L. Han, Nature, 2017, 550, 92. 